Grand Valley State University

ScholarWorks@GVSU

7-27-2015

\title{
mHealth SMS Text Messaging Interventions and to Promote Medication Adherence: An Integrative Review
}

\author{
Tracy DeKoekkoek \\ Michigan State University \\ Barbara Given \\ Michigan State University \\ Charles W. Given \\ Michigan State University \\ Kimberly Ridenour \\ Michigan State University \\ Monica Schueller \\ Michigan State University
}

See next page for additional authors

Follow this and additional works at: https://scholarworks.gvsu.edu/kcon_articles

Part of the Medicine and Health Sciences Commons

\section{ScholarWorks Citation}

DeKoekkoek, Tracy; Given, Barbara; Given, Charles W.; Ridenour, Kimberly; Schueller, Monica; and Spoelstra, Sandra L., "mHealth SMS Text Messaging Interventions and to Promote Medication Adherence: An Integrative Review" (2015). Peer Reviewed Articles. 24.

https://scholarworks.gvsu.edu/kcon_articles/24

This Article is brought to you for free and open access by the Kirkhof College of Nursing at ScholarWorks@GVSU. It has been accepted for inclusion in Peer Reviewed Articles by an authorized administrator of ScholarWorks@GVSU.

For more information, please contact scholarworks@gvsu.edu. 
Authors

Tracy DeKoekkoek, Barbara Given, Charles W. Given, Kimberly Ridenour, Monica Schueller, and Sandra L. Spoelstra

This article is available at ScholarWorks@GVSU: https://scholarworks.gvsu.edu/kcon_articles/24 


\section{JOURNAL OF CLINICAL NURSING}

\section{TITLE PAGE}

Title: mHealth SMS Text Messaging Interventions and to Promote Medication Adherence: An Integrative Review

Concise Title: Adherence and Text Messaging

Authors: Tracy DeKoekkoek, RN; Barbara Given, PhD, RN, FAAN; Charles Given, PhD; Kimberly Ridenour, Undergraduate Nursing Student; Monica Schueller, BA; Sandra L. Spoelstra, PhD, RN

\section{Authors Affiliation:}

Tracy DeKoekkoek, Barbara Given, Kimberly Ridenour, and Sandra Spoelstra

Michigan State University College of Nursing, East Lansing MI

Charles Given

Michigan State University College of Human Medicine, East Lansing MI

\section{Corresponding Author:}

Tracy DeKoekkoek, RN, PhD Student and Research Assistant Michigan State University College of Nursing

Address: 1355 Bogue St., \#C300 East Lansing, MI 48824

Phone: (269) 509-6484

Email: dekoekko@msu.edu

Second author: $\quad$ Barbara Given, PhD, RN, FAAN

Michigan State University College of Nursing

1355 Bogue, Room 383, East Lansing, MI USA 48824

Phone: 517-432-9159 Fax: 517-353-8612

Email Address: barb.given@hc.msu.edu

Third author: Charles W. Given, $\mathrm{PhD}$

Michigan State University

Dept. of Family Medicine, Institute for Health Policy

965 Fee Rd., Room A135 East Fee

East Lansing, MI USA 48864

Phone: 517-353 8131 Fax: 517-432-9977

Email Address: Bill.Given@hc.msu.edu 


\begin{abstract}
Aims and objectives. This article is an integrative review of the evidence for mobile health Short Message Service text-messages as an innovative and emerging intervention to promote medication adherence. Authors completed this review to draw conclusions and implications towards establishing a scientific foundation for use of text-messages to promote medication adherence, thus informing clinical practice.

Background. The World Health Organization has identified medication adherence as a priority global problem. Text-messages are emerging as an effective means of improving health behaviors and in some diseases to promote medication adherence. However, a gap in the literature indicates lack of evidence in guiding theories and content of text-messages, which should be synthesized prior to use in clinical practice.

Design. Integrative review.

Methods. CINAHL, EMBASE, Scopus, the Cochrane Library, and PubMed were searched for relevant studies between 2004 and 2014. Inclusion criteria required interventions testing textmessages to improve medication adherence to prescription oral medications. Articles were assessed for quality of methodology and measures of adherence. An integrative review process was used to perform analysis.

Results. Thirteen articles meeting the inclusion criteria are included in this review. Nine of 13 studies found adherence rates improved between $15.3-17.8 \%$ when using text-messages to promote medication adherence. Text-messages that were standardized, tailored, one- or two-way, and timed either daily to medication regimen, weekly, or monthly showed improvement in medication adherence.

Conclusions. This review established a scientific basis for text-messages as an intervention to improve medication adherence across multiple diseases. Future large rigorous randomized trials are needed to further test text-messaging interventions.

Relevance to clinical practice. This review provides clinicians with the state of the science in regard to text-messaging interventions that promote medication adherence. A description of intervention components are provided to aid nurses in development of text-messages and in translating evidence into practice.
\end{abstract}

Keywords: text messages; SMS; mHealth; medication adherence; intervention; integrative review

Summary box:

This paper contributes to the wider global clinical community in the following manner.

- Reporting on how SMS text messages promote medication adherence rates.

- Providing information for nurses regarding development and content of SMS text messages to promote medication adherence. 


\section{INTRODUCTION}

A meta-analysis of 50 years of research across various conditions and prescribed regimens found a medication adherence rate of 75.2\% (DiMatteo 2004). Another review found adherence rates were $50 \%$ in those who self-administered medications (Haynes et al. 2008). Additionally, $50 \%$ of people with chronic conditions discontinue medications within the first six months prescribed (World Health Organization [WHO] 2003, Centers for Disease Control and Prevention [CDC] 2013). As a consequence, medication adherence is a crucial clinical problem compromising the ability to treat the disease as intended, potentially leading to poor health outcomes (DiMatteo 2004, van Dulmen et al. 2007).

\section{Impact of non-adherence}

Medication adherence impacts both individual health outcomes and healthcare cost (WHO 2003). For example, less than $25 \%$ of those treated for hypertension achieve a healthy blood pressure due to poor medication adherence; and un-controlled hypertension increases risk of ischemic heart disease, stroke, and myocardial infarction. The impact on healthcare cost is significant considering the high rate of chronic conditions treated with medications. For instance, on average, those diagnosed with type 2 diabetes have 1.5 times higher healthcare costs per capita compared to the general population (WHO 2003). There is much to be gained in individual health outcomes and healthcare cost by improving medication adherence.

\section{Defining medication adherence}

A general definition of medication adherence is the extent to which a person follows the medication prescription. For the purpose of this article, medication adherence was defined as following the prescribed regimen, which included timing, frequency, route, and dose (Haynes et al. 2008). 


\section{Factors that influence medication adherence}

Research reveals that older individuals have routines that lead to better adherence outcomes (Krousel-Wood et al. 2009), while other studies show errors of omission and high rates of non-adherence in those who are older (Brown \& Park 2003). Males and non-Caucasians have also been found to be less adherent (Conn et al. 2009). Thus, age, sex, and race may influence adherence.

Depression is known to influence medication adherence. For example, when directly compared, those who were depressed were three times more likely to have poor adherence to prescribed treatment than those who were not depressed (DiMatteo et al. 2000).

Both medication frequency and complexity are also known to influence adherence. A large meta-analysis of medication adherence articles concluded that adherence was adversely proportional to the prescribed medication-taking frequency (Conn et al. 2009).

Other known factors that may influence adherence include adverse effects of the medication, forgetfulness, beliefs about importance of taking the medication, or inability to pay (Haynes et al. 2008). In addition to these, several other factors are evident in the literature. In sum, medication adherence is a complex construct which may be influenced by many factors.

\section{Evidence on improving medication adherence}

Extensive literature exists regarding interventions that improve medication adherence. However, a Cochrane review found that most interventions did not result in significantly improved adherence rates (Haynes et al. 2008). Most of the studies in the review were of short duration, did not include clinical outcomes, or failed to measure medication persistence (Haynes et al. 2008). As a result of these limitations, the global scale of the problem of non-adherence, and costs associated, developing innovative ways to enhance adherence is extremely important. 


\section{A potential solution: mHealth SMS text messages to promote medication adherence}

Short Message Service (SMS) text messages (TMs) are one of the most accessible and common forms of mobile health (mHealth) communication, and evidence is emerging on use of TMs for a wide range of health and behavioral problems (Patrick et al. 2008). TMs may be an effective vehicle to promote medication adherence, as they can be integrated into daily life and adapted to individual needs in real time (Muench et al. 2013). TMs may be one solution to the global health problem of medication adherence.

Six billion people worldwide have access to a cell phone (Pew 2013); and over 98\% of cell phones have TM capability (Cole-Lewis \& Kershaw 2010). A recent Pew Research Report (2013) provides new information regarding cell phone use in the United States (US). In the US, $90 \%$ of adults have a cell phone; and $58 \%$ of those are smartphones. In addition, $67 \%$ of cell phone owners report checking the phone for messages, alerts, or calls even when the cell phone did not ring or vibrate. Also, $44 \%$ slept with the cell phone next to the bed because they did not want to miss calls, TMs, or other updates. Most importantly, $62 \%$ of adults reported using a cell phone for "just-in-time" activities, similar to how TM interventions may be deployed.

In regard to use of TMs to impact health behavior, a recent review of 12 trials between 2005 and 2009 that focused on interventions for disease prevention or management across diseases found TMs consistently improved health behaviors (Cole-Lewis \& Kershaw 2010). In the area of health promotion, evidence exists on using TMs as a reminder for mammograms (Lakkis et al. 2011) and to promote smoking cessation (Severi et al. 2011). With disease management, a study of adult diabetics found that participants were high users of TMs; and the TM intervention improved health outcomes (Ferrer-Roca et al. 2004). 
In summary, TMs are highly accessible, a widely used means of communicating, and are already used in in some health conditions to improve outcomes. While emerging evidence demonstrates TM interventions improve some health outcomes, synthesizing evidence to inform clinical practice remains a challenge.

\section{AIMS}

To date, individual studies have demonstrated TM interventions improved medication adherence rates in some conditions. The aim of this review was to compare mHealth SMS TM interventions that promoted medication adherence. Objectives were: (1) to report and summarize evidence of TM interventions to promote medication adherence; and (2) to report TM components which improved medication adherence rates. The intent was to synthesize for translation of evidence to be applied in clinical practice.

\section{METHODS}

\section{Integrative review}

The method of integrative review as described by Whittemore and Knafl (2005) was used for this review article, to include all levels of evidence. An integrative review is a comprehensive process that involves data collection and extraction of data to uniquely summarize existing literature not limited to randomized control trials with the aim to contribute directly to evidencebased practice (Whittemore \& Knafl 2005). The process of identifying articles was conducted in two steps. First, electronic databases were searched for pertinent articles published over the past 10 years. Second, the reference lists of identified articles were searched for relevant studies.

\section{Search strategies}

Informed by these review steps, the literature was investigated for published articles on TM interventions to promote medication adherence. A search in Cumulative Index to Nursing 
and Allied Health Literature (CINAHL), Excerpta Medica dataBASE (EMBASE), Scopus, the Cochrane Library, and PubMed was conducted using keywords "text message" or "text messaging" or "text" and "medication adherence" and "intervention." The search was limited to English-language peer reviewed articles published between 2004 and 2014. As shown in the Figure, 55 articles were retrieved on TMs using keywords through database search. References of those articles were also reviewed for relevant articles.

\section{Inclusion and exclusion criteria}

The Preferred Reporting Items for Systematic Reviews and Meta-Analyses (PRISMA) protocol was used for this review (http://www.prisma-statement.org/). Inclusion criteria were defined, including: (1) English-language, peer reviewed articles published from 2004 to 2014; and (2) articles containing TMs used as an intervention to improve rates of medication adherence to prescription pills in adults (18 years or older). Exclusion criteria consisted of: (1) articles that did not include results of medication adherence rates; (2) medications were not in pill form (e.g., insulin injections, inhalers, or topical); (3) the TM used a picture rather than words; or (4) the TM interventions were bundled with other interventions.

\section{Data extraction and analysis}

Data on study characteristics, intervention characteristics, and results pertaining to the effects of the TM interventions on medication adherence rates were summarized from the eligible articles. Data extraction was performed by four reviewers. First, two reviewers independently read the articles carefully and documented relevant information in a table. Second, the two other reviewers confirmed the accuracy of the information. This iterative process conducted on the articles meeting the inclusion criteria continued until consensus was reached. 


\section{Messaging in TM interventions}

There is a wide variation in TMs in the literature. For this synthesis, two categories of messaging were described. The first categorization regards content: 1) TMs with message content that was standardized; and 2) TMs with messages that had content tailored to the individual. The second set of categories was: 1) one-way TMs with messages sent to the individual; and 2) two-way TMs, with messages sent to the individual and the individual sends a return TM.

\section{RESULTS}

Thirteen studies were found employing a TM intervention to promote medication adherence to prescribed oral medications (see Table). Articles included 1 Cochrane review, 8 randomized control trials (RCT) studies, 3 quasi-experimental design, and 1 secondary data analysis. The following describes each study.

\section{Study characteristics}

Articles in this review were published between 2004 and 2014. All interventions were implemented in a community setting. Seven of the studies took place in the United States. The remaining studies took place in Brazil, Cameroon, Iran, Malaysia, Kenya, and Spain.

\section{Participant characteristics}

Sample size ranged from 21 to 704 participants; with a total 2,463 participants. Age varied from 18 to 83 years of age; with a mean of 47.5 years old ( $n=10$ studies reporting) in this review. Overall, $55 \%$ were female ( $n=11$ studies reporting). Of the 7 studies reporting race or ethnicity, 34\% were Caucasian, 20\% African Americans, 24\% Malaysians, and less than 5\% each Chinese, Indian, or Hispanic. 
TM interventions were targeted toward various populations. People with a human immunodeficiency virus (HIV) diagnosis prescribed antiretroviral therapy (ART) were the most prevalent $(46.2 \%, \mathrm{n}=5)$; followed by schizophrenia $(15.4 \%, \mathrm{n}=2)$. In addition, one study in each of the following diagnosis was found (7.9\% each): diabetes, coronary heart disease, epilepsy, and any chronic disease diagnosis. Finally, there was 1 study in those prescribed an antibiotic after Emergency Department discharge (7.9\%); and another in oral contraception use (7.9\%).

\section{Training participants to TM}

One article reported training participants in a lab setting using a 30-minute PowerPoint presentation followed by 3 trials of receiving and sending TMs and a 10-minute in-home visit to retrain during the initial days of the study to assure ability to TM (Granholm et al. 2012). Another study provided instructions to participants on how to use a cell phone and assessed ability to read TMs (Zolfaghari et al. 2012).

\section{Development of TM content}

Only 3 of the 13 studies reported using a theoretical model as a basis for designing TM content. One study reported designing TMs using the health belief model (Mbuagbaw et al. 2012), a second used Self-Efficacy Theory (Park et al. 2014), and a third used an un-named behavioral learning theory (Montes et al. 2012).

Two studies reported detailed information on how TM content was developed. One study developed messages based on evidence from a systematic review in those with HIV using content experts and tested in 8 participants with HIV prior to finalizing messages (Lewis et al. 2013). Another study developed messages based on data from focus groups using the health 
belief model with an emphasis on motivational content (Mbuagbaw et al. 2012). Variations in approaches among the studies will be discussed.

Test text messages. One study sent a test TM to all participants: "Welcome to the [antibiotic name] study. Text back 'yes' when you have picked up your prescription” (Suffoletto et al. 2012). Participants were expected to respond within 6 hours, if they did not respond, a second TM was sent: "We're concerned you have not picked up your prescription for [antibiotic]. Text us 'yes' when you do.” This TM was repeated 4 times (Suffoletto et al. 2012).

Standardized and tailored text message interventions. Nine studies used standardized TM content while 4 studies tailored the TM content based on assessment data or participant choice (see Table). The 9 standardized TM studies delivered the same message content without variation to participants. For example: "Please remember to take your birth control pill" was delivered daily to each participant (Hou et al. 2010). In 4 of the standardized content TM studies, participants were allowed some degree of control over choosing the timing of when TMs were delivered (da Costa et al. 2012, Foreman et al. 2012, Horvath et al. 2012, Montes et al. 2012).

The 4 tailored TM studies delivered content with variation (Hardy et al. 2011, Granholm et al. 2012, Lewis et al. 2013, Park et al. 2014). Tailored TMs were most often individualized based on assessment. One HIV study tailored TMs after conducting the baseline assessment of medication non-adherence in the past 7 days (Lewis et al. 2013). For those who were nonadherent, TMs were delivered daily, timed to the medication regimen; and for those who were assessed as adherent, weekly TMs were sent to encourage adherence to the medication as prescribed. TMs sent to non-adherent participants stated: "Stop, drop, and pop. Take your meds now." TMs sent to adherent participants stated: "He shoots! He scores! Perfect med adherence. Great Job!" In a second study using tailored TMs, the following message was used: "But you 
said taking meds helped you" followed by an insertion of a tailored comment based on the assessment of what the individual felt was a personal benefit of taking the medication (Granholm et al. 2012). Another study tailored TM content based on participant interests such as sports news, weather, or famous quotes (Hardy et al. 2011).

One-way and two-way text message interventions. As shown in the Table, variations in one-way and two-way TMs were also found. Seven studies had one-way intervention design, sending a TM to the participant, but not requiring a return TM response. The remaining 6 studies used a two-way TM intervention design, sending a TM to the participant and requiring a TM response from the participant.

In regard to two-way TMs, 1 study measured satisfaction with the intervention in the response TM (Granholm et al. 2012). Another study used two-way TMs response to address needs or questions that the participants identified (Zolfaghari et al. 2012).

Two studies that used two-way TMs reported on TM response rates. One found a median TM response rate of $78 \%$, with the odds of a response TM declining by 0.51 (95\% confidence interval [CI] 0.27-0.94) between the first and second week of the intervention (Hardy et al. 2011). A second study found a median TM response rate of $86 \%$ over the 12 weeks of the study (Granholm et al. 2012).

\section{Implementation of TM interventions}

Twelve of the 13 articles report some details regarding the implementation process of sending TMs. Eleven protocols included utilization of a third-party web-based platform to send and receive TMs. Two studies specified that one study staff person (nurse or secretary) sent the TMs to each study participant. Three studies mentioned quality assurance processes to ensure accuracy of the TMs. Only one (Lester et al., 2010) provided an estimate of cost per TM, which 
was $\$ 0.05$ per TM or about $\$ 20$ per 100 participants per month. Three studies reported some information on the burden of implementing the intervention in consideration to workload. In addition to normal outpatient staffing, one doctor and one nurse were hired to adjust for workload (da Costa et al, 2012). Another study estimated that one clinical nurse could safely manage TM reminders for approximately 1000 patients (Lester et al., 2010). Finally, one study estimated without statistically evidence that TM reminders were less expensive and less time consuming than usual follow-up phone calls (Zolfaghari et al. 2012).

\section{Dose of TM interventions}

The dose of the TM intervention varied widely, from 3 TMs per day over 12 weeks (Lewis et al. 2013) to once-per-month TMs for 3 months (Lua \& Neni 2013). Among the 13 studies, 8 studies sent daily TMs, 1 study sent TMs two or three times a day, 2 studies sent TMs less than daily but more than once-per-week, 1 study sent weekly TMs, and 1 study sent monthly TMs (see Table). One study compared efficacy of weekly and daily TMs and found that weekly messages were more likely to be associated with higher adherence rates (Horvath et al. 2012).

\section{Measures of medication adherence}

Various measures of medication adherence were used among the studies. In 6 studies, a single measure of adherence was used. The Cochrane Review included 2 studies: 1 used selfreport and the other used a Medication Event Monitoring System (MEMS), a device that records the day and time that each medication bottle was opened (Horvath et al. 2012). Two studies used self-report of adherence by TM response (Granholm et al. 2012, Suffoletto et al. 2012). Another study used a visual analog scale (Mbuagbaw et al. 2012). One study used the Morisky adherence tool to measure adherence rates by self-report (Lua \& Neni 2013). Finally, prescription refills 
from claims were used in 1 study to calculate the proportion of days covered to measure adherence rates (Foreman et al. 2012).

Three studies used 2 measures of medication adherence. Two used an electronic device and written diary and 1 used a blood draw for the medication level and self-report (Hou et al 2010, Zolfaghari et al. 2012, Lewis et al. 2013).

The remaining 4 studies used 3 measures of medication adherence (Hardy et al. 2011, da Costa et al. 2012, Mbuagbaw et al. 2012, Park et al. 2014). Two articles report using the combination of pill count, MEMS, and self-report. Another used self-report, a visual analog scale, and prescription refill data, and the fourth used MEMS, two way TMs, and self-report using the Morisky Medication Adherence scale. Two of these studies compared the measures and found no statistical difference between reported medication adherence rates (da Costa et al. 2012, Mbuagbaw et al. 2012). However, 1 study that compared adherence measures found a difference between MEMS, pill count, and self-report, reporting that MEMS was the most reliable (Hardy et al. 2011).

\section{Effectiveness of TM interventions to improve rates of medication adherence}

Nine of the 13 studies found higher rates of medication adherence using a TM intervention. Each will be discussed.

Three studies in participants with HIV ART treatment found higher rates of adherence using TMs. A meta-analysis found weekly TMs were associated with lower rates of nonadherence at 48 to 52 weeks (RR 0.78, CI 0.68 to 0.89 ) (Horvath et al. 2012). One RCT found adherence rates were higher in the TM group at week $3(28.1 ; \mathrm{p}=0.01)$ and at week 6 (33.4; p <0.001) (Hardy et al. 2011). A cohort study found $68 \%$ of participants improved or remained adherent throughout the trial (Lewis et al. 2013). That same study examined viral load and 
cluster of differentiation (CD4) as measures of adherence to ART. Viral load is the extent to which HIV is present and CD4 is a glycoprotein found on the surface of immune cells, which indicates a functioning immune system. Lab results indicated a decline, although not statistically significant, in viral load $(\mathrm{p}=0.12)$ and a significant increase in CD4 counts $(\mathrm{p}=0.037)$ after three months (Lewis et al. 2013).

Two studies conducted in participants with schizophrenia report that TMs improved medication adherence rates. One study found an overall high adherence rate over the 12 week intervention (Granholm et al. 2012). The second found adherence was significantly better in the TM group at $3(\mathrm{p}=0.02)$ and $6(\mathrm{p}=0.04)$ months (Montes et al. 2012).

Four studies were in various chronic conditions. The RCT in participants with coronary heart disease, which examined antiplatelet adherence rates, found a higher percent of correct doses taken $(\mathrm{p}=0.02)$, number of doses $(\mathrm{p}=0.01)$, and percent of doses taken on schedule $(\mathrm{p}=0.01)$ in the TM group compared to the control group (Park et al. 2014). A trial in participants with diabetes found adherence rates $(\mathrm{p}<0.001)$ and hemoglobin $\mathrm{A} 1 \mathrm{C}(\mathrm{p}<0.01)$ improved between pre- and post-test over 3 months (Zolfaghari et al. 2012). A study of participants with epilepsy found adherence was significantly higher with TMs (p<0.001) (Lua \& Neni 2013). Finally, in a study in multiple chronic conditions, the TM group had higher rates of adherence than the control group (85\% compared to $77 \%$, p<0.001) (Foreman et al. 2012).

Four of the 13 studies did not find higher rates of medication adherence using TM interventions. One RCT in participants with HIV taking ART, found no increase in the rate of adherence for participants receiving TMs (p=0.54) (Mbuagbaw et al. 2012). In a second study looking at HIV, participants self-reported adherence rates for the control group were $84.6 \%$ while the TM group were 100\%, which was not statistically significant (p>0.05) (da Costa et al. 
2012). The study on oral contraceptive medication adherence rates found no difference using the number of missed pills per cycle (intervention $4.9 \pm 3.0$ control $4.6 \pm 3.5 ; \mathrm{p}=0.60$ ) (Hou et al 2010). Finally, no statistical difference was found in an RCT examining adherence to antibiotics after discharge from the emergency department between the TM intervention group and the control group ( $\mathrm{p}=0.1)$ (Suffoletto et al. 2010).

\section{Satisfaction with TM intervention}

Five of 13 studies examined satisfaction with TM interventions. Each found moderate to high levels of helpfulness from the TMs to promote medication adherence (da Costa et al. 2012, Granholm et al. 2012, Mbuagbaw et al. 2012, Suffoletto et al. 2012, Lewis et al. 2013). One study reported $93 \%$ of participants always read the TMs (Lewis et al. 2013). The study conducted by da Costa et al. (2012) included a detailed satisfaction analysis. In the da Costa (2012) article, 63.6\% felt TMs assisted with adherence and 45.5\% said TMs provided incentive to take medication. In this study, TMs were not delivered at the time the medication was to be taken; however, $27.7 \%$ requested delivery of the TMs closer to the time the medication was to be taken. In regard to intervention dose, $90.9 \%$ wanted to continue with TMs when the 4 month intervention was complete. In the Sufoletto et al. (2013) study, 94\% of antibiotic takers discharged from the emergency department said that they would use a TM reminder again.

\section{Confidentiality and privacy issues}

Participant comfort with privacy was reported in 4 of the 13 articles. One study guaranteed confidentiality to participants by password protecting TMs (Zolfaghari et al. 2012). Two studies reported on participants who withdrew from the study due to confidentiality concerns (Mbuagbaw et al. 2012, Park et al. 2014). In one HIV article, participants agreed that 
they did not want the word "medication" included in the content of the TM, but felt that receiving a TM would not jeopardize their privacy (Hardy et al. 2011).

\section{DISCUSSION}

This review demonstrated that TMs interventions were effective at improving medication adherence rates from 15.3 to $17.8 \%$ across multiple diseases or conditions. However, there is still much to be learned about TM interventions.

Few studies were built on theoretical underpinnings or conceptual frameworks, and evidence is emerging on the importance of theory driven TM content (Muench et al. 2013). Based on the varying measures of adherence and types of TM designs, determining if tailored or standardized TMs were more effective was not possible in this review. Evidence is emerging, that in some conditions, tailored interventions are a more effective approach to achieve behavior change (Noar et al. 2007). This review had nearly equal one-way and two-way TM interventions. However, we were unable to discern which mode was more effective, identifying a gap in the science. The choice of whether to use one-way or two-way TMs may be based on type of disease or medication, motivation level, self-efficacy, or user preference. Limited results and variations restricted further conclusions regarding efficacy of TM dose—daily, weekly, or monthly or the timing of TMs (e.g., timed to the daily medication regimen administration).

The study by Park et al. (2014) supported a TM intervention in older adults. Researchers developing future TM reminder studies might consider including informal caregivers, as no articles discussed caregiver support. Informal caregiver support may be especially important when enrolling older adults with chronic conditions, who often have family members helping with care. 
There are important precautions that can be taken in order to minimize risks associated with communicating to participants via TMs. One suggestion regarding privacy considerations is to obtain participant authorization to send TMs that could contain personal health information (Harris et al. 2013). Another step for protecting participants is to suggest that they password protect mobile phones to limit access to the information (Harris et al. 2013). These precautions should reduce confidentiality and privacy issues.

In summary, there are many aspects of a TM intervention that should be considered when using TMs in clinical practice. Overall, among these studies, TMs were demonstrated to be easy to use and an effective means to promote medication adherence across many disease types, as indicated by the wide variation of participant diagnoses and medication type, for those enrolled in the 13 studies. This review also found high satisfaction of TMs as an intervention to improve medication adherence. Clinicians should consider content (standardized or tailored) of TMs and timing of delivery, such as just-in-time for the medication dose, meal time, or morning, afternoon, and night. Further, the dose or how many messages are delivered, such as daily, weekly, or monthly, should be considered. Depending on the goal of the intervention and the desired use, it is important to consider potential outcomes related to one-way or two-way TMs. Further research should be conducted regarding using two-way TMs as a potential way to effectively measure medication adherence.

\section{Limitations}

This synthesis entails some limitations due to the state of the science. There is no standard measure of medication adherence, making comparisons across studies difficult. Some studies used self-report as the only means of measuring adherence, and thus, medication adherence may have been over-reported. Many of these are single diagnosis studies and did not 
assess for comorbidities, which limits generalizability. Most of the studies were very short in duration; consequently, the effectiveness of TMs over time could not be reported. Other limitations include the lack of discussion regarding confidentiality and workload burden when using TMs. Many of the articles included very thorough discussion of the content of the TMs; however, very little detail was provided on the execution of the intervention such as if a software platform was used to send/ receive TMs, cost of the TMs, or if the researchers found the TM process to be burdensome.

\section{RELEVANCE TO CLINICAL PRACTICE}

If technology were readily available in clinical settings, TMs could be used as an intervention to promote medication adherence. Forgetfulness, one of the main reasons for nonadherence (Conn et al. 2009), could be prevented if TMs were used to remind individuals to take their medications as prescribed. TMs as an intervention to promote medication adherence can include content to address some of these modifiable adherence influencers. For example, reminders could be timed to be delivered on the prescription regimen (e.g., "take your antibiotic [drug name] now") (Puccio et al. 2006). Self-efficacy could be increased by tailoring the content of the reminders to empower individuals to feel capable of managing their medications (Muench et al. 2013). In addition, providing the ability to send a return TM (e.g., took or did not take medication [two-way]), may increase self-efficacy and engage individuals in self-care (Britto $e t$ al. 2011). Participants reported being interested in receiving TM reminders for future prescriptions (Suffoletto et al. 2012). Participants often found TMs less intrusive and simpler than telephone reminders (da Costa et al. 2012). Finally, including interesting facts or illness perception cues in TMs may further engage individuals in their care by increasing the perception for the necessity to adhere to their medication. 
Nurses could use this relatively simple method of medication reminders to have a profound effect on health outcomes through promoting medication adherence. However, it may not be feasible or practical to require individual nurses to send TMs to patients to take medications. Nurses can advocate for patients by requesting that health care systems investigate software platforms or programming to incorporate TMs into care. TMs may be a feasible way for case managers to communicate with patients, but little is known about the burden to clinicians of performing a TM intervention. Prior to wide use in clinical settings, offices and health care systems that are currently employing TM reminders should report evidence regarding burden of intervention, cost of TMs, development of TM content, effect on health outcomes, and process of integrating TMs into care.

\section{CONCLUSIONS}

Medication adherence is a problem of global importance, and innovative interventions such as TM reminders need to be tested to help solve this challenging clinical problem (WHO 2003, CDC 2013). TMs may be a simple-to-use, cost-effective means for nurses to promote medication adherence, and thus assure treatment effectiveness. Improving medication adherence may be the most cost-efficient investment compared to the cost of complications related to nonadherence (WHO 2003).

The majority of these articles support TMs as an effective intervention to promote medication adherence, yet much work needs to be done to understand when and how TM interventions may be effective at improving medication adherence. Future research needs to examine effectiveness of TMs. First, the intervention needs to be examined over a longer period of time to determine if persistence (e.g., completion of full regimen prescribed for course of treatment) occurs. Second, standardized and tailored TMs need to be examined as well as one- 
and two-way TMs. Third, TMs need to be tested in medications with complex regimens and across multiple diseases, age groups, and races. In addition, variation in content or cues for building self-efficacy needs to be explored to determine if they are more effective than generic TMs. TMs have the potential to help promote medication adherence and improving health outcomes. 
Figure CONSORT of articles found on text messaging to improve medication

adherence for this review

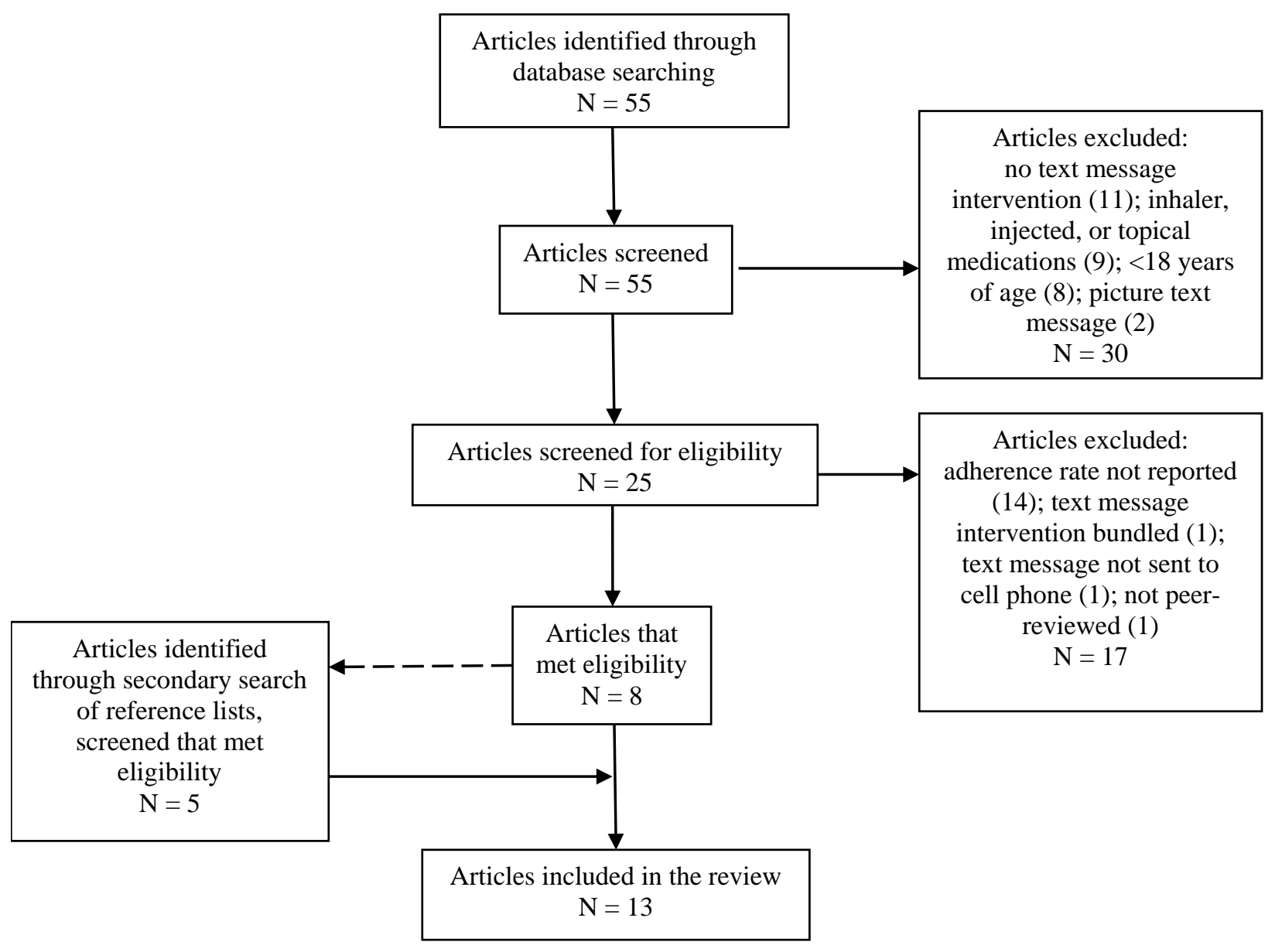




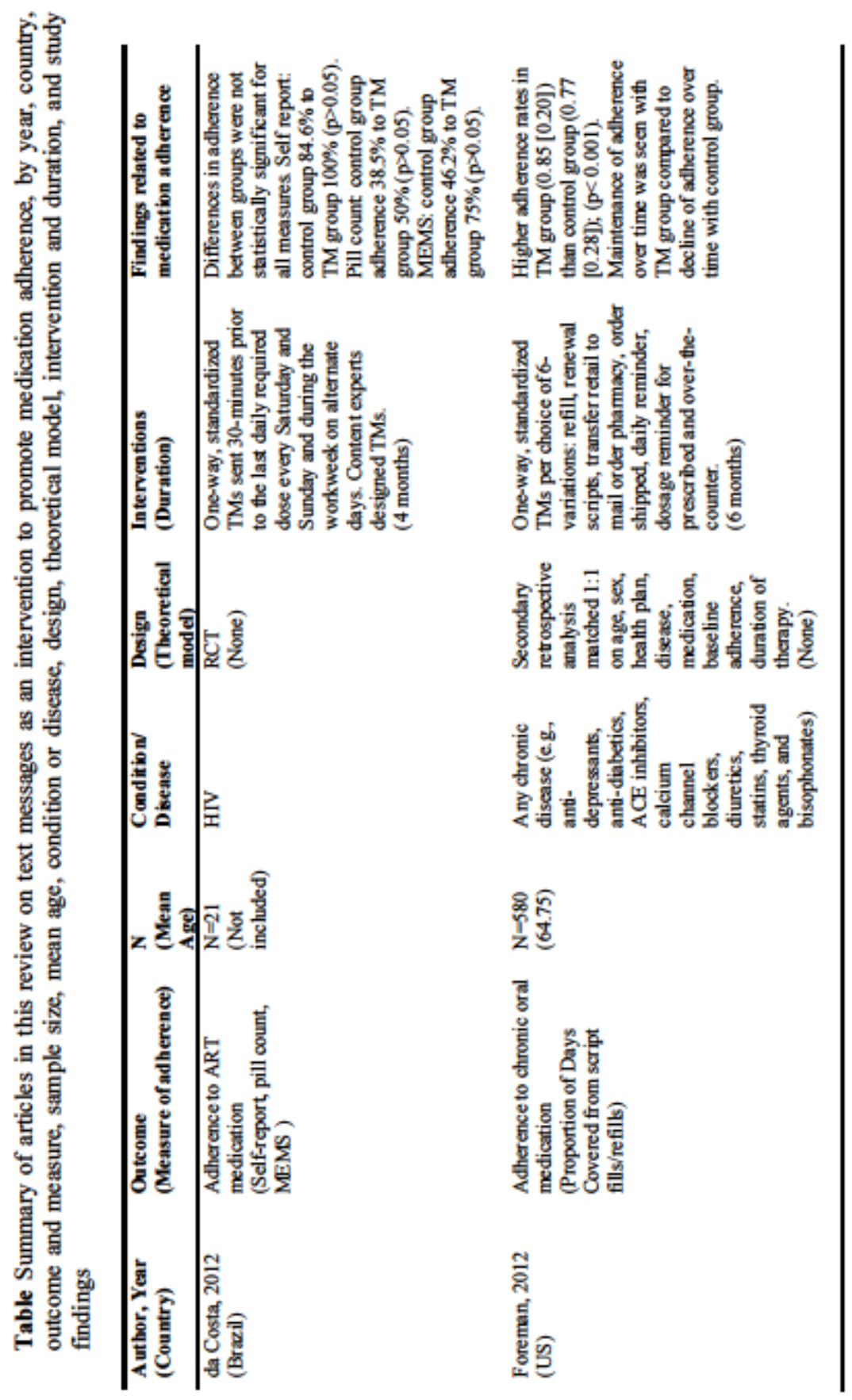




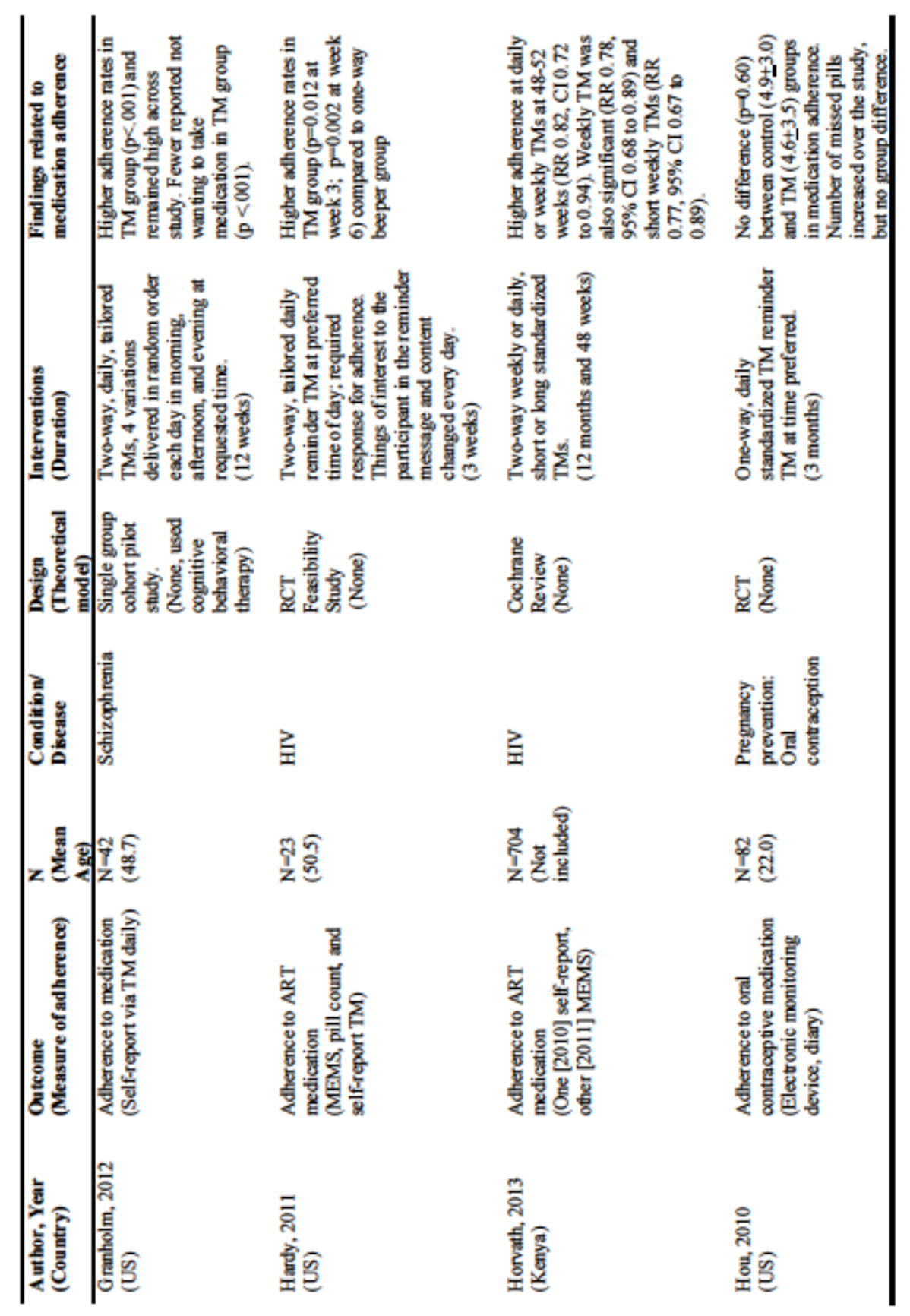




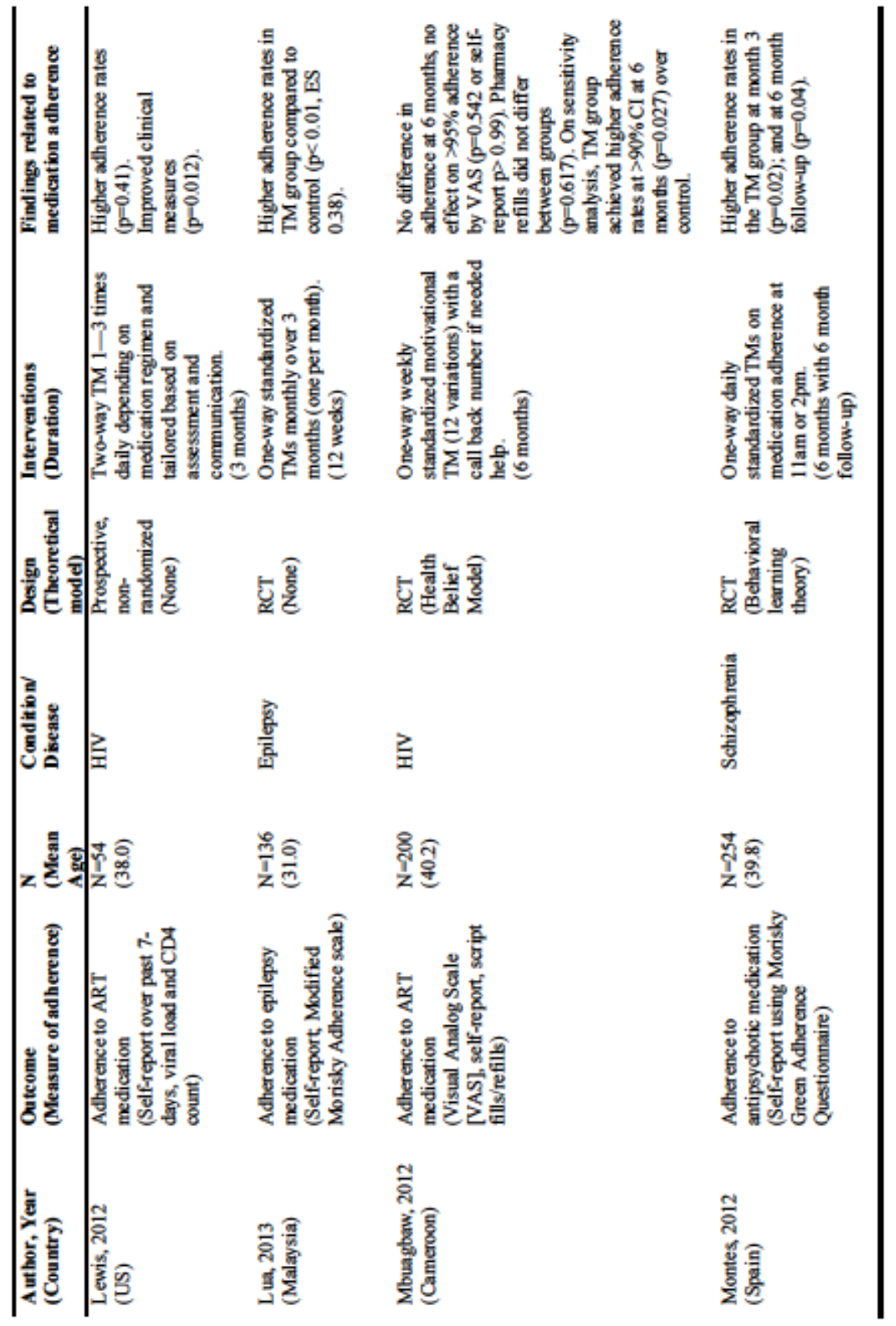




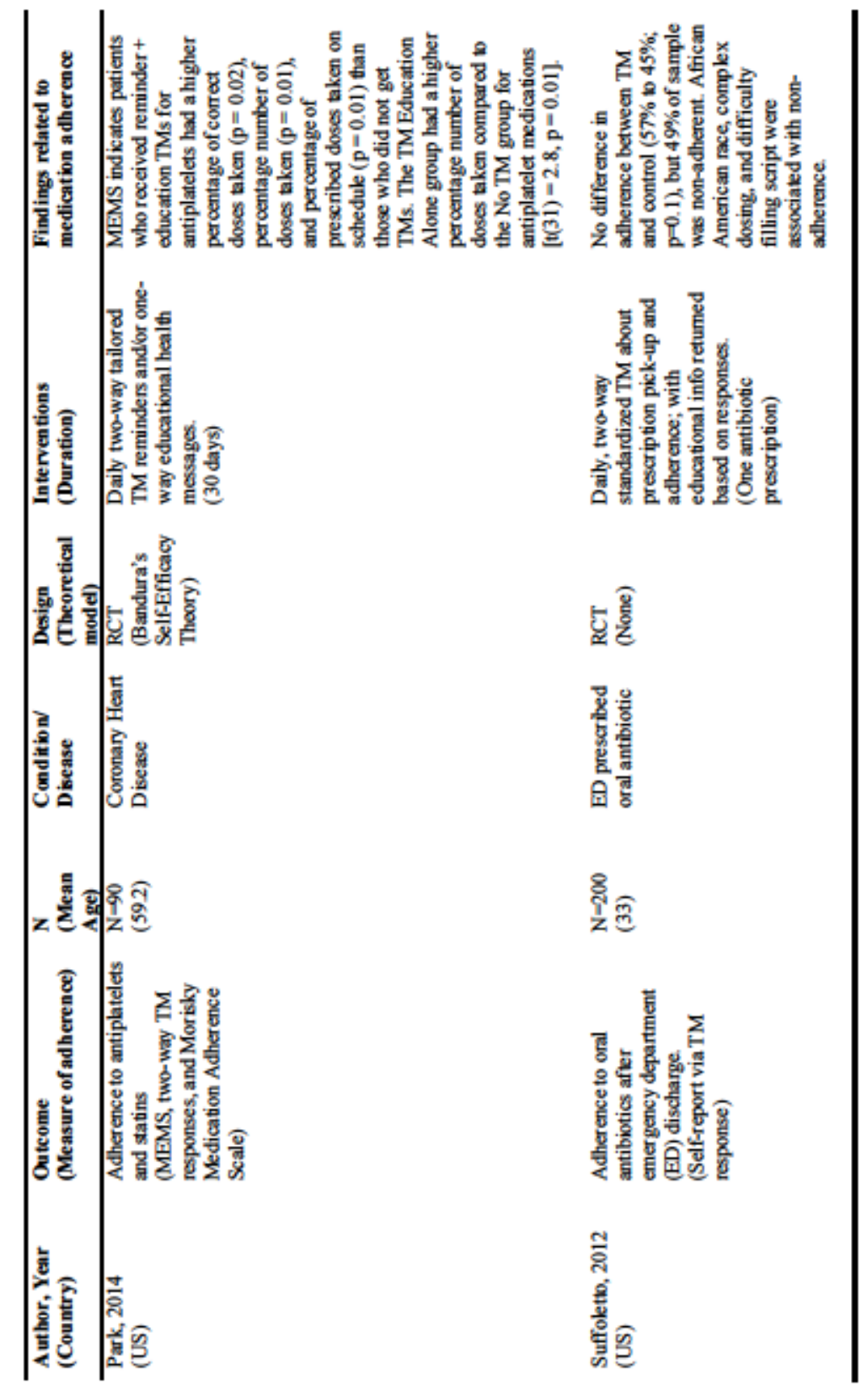




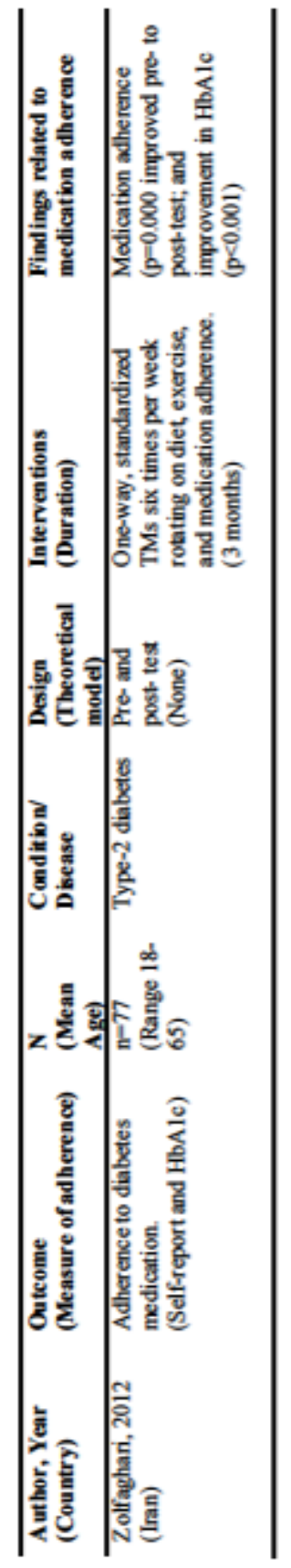




\section{References}

Britto MT, Munafo JK, Schoettker PJ, Vockell ALB, Wimberg JA \& Yi MS (2011) Pilot and feasibility test of adolescent-controlled text messaging reminders. Clinical Pediatrics 51, 114-121. doi: 10.1177/0009922811412950

Brown SC \& Park DC (2003) Theoretical models of cognitive aging and implications for translational research in medicine. Gerontologist 43, 57-67.

Centers for Disease Control and Prevention (2013) Medication adherence. Available at: http://www.cdc.gov/primarycare/materials/medication/

Cole-Lewis H \& Kershaw T (2010) Text messaging as a tool for behavior change in disease prevention and management. Epidemiologic Reviews 32, 56-69. doi: 10.1093/epirev/mxq004

Conn VS, Hafdahl AR, Cooper PS, Ruppar TM, Mehr DR \& Russell CL (2009) Interventions to improve medication adherence among older adults: meta-analysis of adherence outcomes among randomized controlled trials. Gerontologist 49, 447-462. doi: 10.1093/geront/gnp037

da Costa TM, Barbosa BJ, Sigulem D, de Fátima Marin H \& Pisa IT (2012) Results of a randomized controlled trial to assess the effects of a mobile SMS-based intervention on treatment adherence in HIV/AIDS-infected Brazilian women and impressions and satisfaction with respect to incoming messages. International Journal of Medical Informatics 81, 257-269.

DiMatteo MR (2004) Variations in patients' adherence to medical recommendations: a quantitative review of 50 years of research. Medical Care 42, 200-209. doi: $10.1097 / 01$

DiMatteo MR, Lepper HS \& Croghan TW (2000) Depression is a risk factor for noncompliance with medical treatment: a meta-analysis of the effects of anxiety and depression on patient adherence. Archives of Internal Medicine 160, 2101-2107.

Ferrer-Roca O, Cárdenas A, Diaz-Cardama A \& Pulido P (2004) Mobile phone text messaging in the management of diabetes. Journal of Telemedicine \& Telecare 10, 282-285.

Foreman KF, Stockl KM, Le LB, Fisk E, Shah SM, Lew HC, Solow BK \& Curtis BS (2012) Impact of a text messaging pilot program on patient medication adherence. Clinical Therapeutics 34, 1084-1091. doi: 10.1016/j.clinthera.2012.04.007

Granholm E, Ben-Zeev D, Link PC, Bradshaw KR \& Holden JL (2012) Mobile assessment and treatment for schizophrenia (MATS): a pilot trial of an interactive text-messaging intervention for medication adherence, socialization, and auditory hallucinations. Schizophrenia Bulletin 8, 414-425. doi: 10.1093/schbul/sbr155

Hardy H, Kumar V, Doros G, Farmer E, Drainoni ML, Rybin D, Myung D, Jackson J, Backman E, Stanic A \& Skolnik PR (2011) Randomized controlled trial of a personalized cellular phone reminder system to enhance adherence to antiretroviral therapy. AIDS Patient Care and STDs 25, 153-161. doi: 10.1089/apc.2010.0006

Harris JL, Furberg R, Martin N, Kuhns L, Lewis MA, Coomes C, Williams P \& Uhrig JD (2013) Implementing an SMS-based intervention for persons living with human immunodeficiency virus. Journal of Public Health Management \& Practice 19, E916. doi: 10.1097/PHH.0b013e3182582b59 
Haynes RB, Ackloo E, Sahota N, McDonald HP \& Yao X (2008) Interventions for enhancing medication adherence. Cochrane Database Syst Rev 2, CD000011. doi: 10.1002/14651858.CD000011.pub3

Horvath T, Azman H, Kennedy GE \& Rutherford GW (2012) Mobile phone text messaging for promoting adherence to antiretroviral therapy in patients with HIV infection. Cochrane Database of Systematic Reviews 3, 1-39. doi: 10.1002/14651858.CD009756

Hou MY, Hurwitz S, Kavanagh E, Fortin J \& Goldberg AB (2010) Using daily text-message reminders to improve adherence with oral contraceptives: a randomized controlled trial. Obstetrics \& Gynecology 116, 633-640. doi: 10.1097/AOG.0b013e3181eb6b0f

Krousel-Wood MA, Muntner P, Islam T, Morisky DE \& Webber LS (2009) Barriers to and determinants of medication adherence in hypertension management: perspective of the cohort study of medication adherence among older adults. Med Clin North Am 93, 753-769. doi: 10.1016/j.mcna.2009.02.007

Lakkis NA, Atfeh AM, El-Zein YR, Mahmassani DM \& Hamadeh GN (2011) The effect of two types of SMS-texts on the uptake of screening mammogram: a randomized controlled trial. Preventive Medicine 53, 325-327.

Lester RT, Ritvo P, Mills EJ, Kariri A, Karanja S, Chung MH, Jack W, Habyarimana J, Sadatsafavi M, Najafzadeh M, Marra CA, Estambale B, Ngugi E, Ball TB, Thabane L, Gelmon LJ, Kimani J, Ackers M \& Plummer FA (2010) Effects of a mobile phone short message service on antiretroviral treatment adherence in Kenya (WelTel Kenya1): a randomised trial. Lancet 376, 1838-1845. doi: 10.1016/S01406736(10)61997-6

Lewis MA, Uhrig JD, Bann CM, Harris JL, Furberg RD, Coomes C \& Kuhns LM (2013) Tailored text messaging intervention for HIV adherence: a proof-of-concept study. Health Psychology 32, 248-253. doi: 10.1037/a0028109

Lua PL \& Neni WS (2013) A randomised controlled trial of an SMS-based mobile epilepsy education system. Journal of Telemedicine and Telecare 19, 23-28. doi: $10.1177 / 1357633 X 12473920$

Mbuagbaw L, Thabane L, Ongolo-Zogo P, Lester RT, Mills EJ, Smieja M, Dolovich L \& Kouanfack C (2012) The Cameroon Mobile Phone SMS (CAMPS) trial: a randomized trial of text messaging versus usual care for adherence to antiretroviral therapy. PLoS One 7, e46909. doi: 10.1371/journal.pone.0046909

Montes JM, Medina E, Gomez-Beneyto M \& Maurino J (2012) A short message service (SMS)-based strategy for enhancing adherence to antipsychotic medication in schizophrenia. Psychiatric Research 200, 88-95. doi: 10.1016/j.psychres.2012.07.034

Muench F, Weiss RA, Kuerbis A \& Morgenstern J (2013) Developing a theory driven text messaging intervention for addiction care with user driven content. Psychology of Addictive Behaviors 27, 315-321. doi: 10.1037/a0029963

Noar SM, Benac CN \& Harris MS (2007) Does tailoring matter? Meta-analytic review of tailored print health behavior change interventions. Psychological Bulletin 133, 673693. doi: 10.1037/0033-2909.133.4.673

Park LG, Howie-Esquivel J, Chung ML \& Dracup K (2014) A text messaging intervention to promote medication adherence for patients with coronary heart disease: a randomized controlled trial. Patient Education and Counseling 94, 261-268. doi: 10.1016/j.pec.2013.10.027 
Patrick K, Griswold WG, Raab F \& Intille SS (2008) Health and the mobile phone. American Journal of Preventatiave Medicine 35, 177-181.

Pew Research Internet Project (2013). Mobile Technology Fact Sheet. Found at http://www.pewinternet.org/fact-sheets/mobile-technology-fact-sheet/

Puccio JA, Belzer M, Olson J, Martinez M, Salata C, Tucker D \& Tanaka D (2006) The use of cell phone reminder calls for assisting HIV-infected adolescents and young adults to adhere to highly active antiretroviral therapy: a pilot study. AIDS Patient Care \& STDs 20, 438-444.

Severi E, Free C, Knight R, Robertson S, Edwards P \& Hoile E (2011) Two controlled trials to increase participant retention in a randomized controlled trial of mobile phonebased smoking cessation support in the United Kingdom. Clinical Trials 8, 654-660. doi: 10.1177/1740774511416524

Suffoletto B, Calabria J, Ross A, Callaway C \& Yealy DM (2012) A mobile phone text message program to measure oral antibiotic use and provide feedback on adherence to patients discharged from the emergency department. Academic Emergency Medicine 19, 949-958.

Uhrig JD, Lewis MA, Bann CM, Harris JL, Furberg RD, Coomes CM \& Kuhns LM (2012) Addressing HIV knowledge, risk reduction, social support, and patient involvement using SMS: results of a proof-of-concept study. Journal of Health Communications 17, 128-145. doi: 10.1080/10810730.2011.649156

van Dulmen S, Sluijs E, van Dijk L, de Ridder D, Heerdink R \& Bensing J (2007) Patient adherence to medical treatment: a review of reviews. BMS Health Services Research BioMed Central 7, 55.

Whittemore R \& Knafl K (2005) The integrative review: updated methodology. Journal of Advanced Nursing 52, 546-553.

World Health Organization (2003) Adherence to long-term therapies: evidence for action. Available at: http://www.who.int/entity/chp/knowledge/publications/adherence_introduction.pdf

Zolfaghari M, Mousavifar SA, Pedram S \& Haghani H (2012) The impact of nurse short message services and telephone follow-ups on diabetic adherence: which one is more effective? Journal of Clinical Nursing 21, 1922-1931. doi: 10.1111/j.1365-

2702.2011.03951.x 